\title{
MEDICAL TRAINING FOR DISASTER SITUATIONS
}

\author{
by J.-A. Baumann
}

We take pleasure in publishing some long excerpts from an article which appeared in the Revue suisse de médecine militaire (Basle, December 1972) and which that review has very kindly permitted us to reproduce. The author, a colonel in the Swiss army medical corps and head physician of a territorial zone, gives his personal views on medical practice in disaster situations and refresher courses in institutions for the training of medical personnel, specialized auxiliaries and first-aid workers. The subject is one which will undoubtedly interest our readers, for the Red Cross is faced with mass relief problems; and it is in conflicts or as a result of natural disasters that problems of medicine and surgery are of crucial importance, and concern doctors, nurses and first-aid workers, some of whom work under the sign of the red cross, the red crescent or the red lion and sun.

(Editor)

Medical practice in disaster relief has a very specific aspect as compared with medicine in general, but I must point out that the difference lies in its organization rather than in its therapeutic nature. A doctor who has before him a sick or wounded person, or any other patient, will unstintingly provide care according to the knowledge and training he received at medical school. He will do so in accordance with the code of ethics of his calling, inculcated by his teachers. Doctors throughout the world draw inspiration from the Hippocratic Oath, the Prayer of Maimonides ${ }^{1}$ or the Geneva Declaration ${ }^{2}$. All of this has been accepted and need not enter the type of

${ }^{1}$ A Jewish physician and philosopher in 12th-century Spain. He was the type of doctor who could transcend nationality and religion, rise above the social and political conditions of the day, accept the truths of eternal philosophy and "invoke God" as all men can do.

2 A modern code established by the World Medical Association in 1948. 
training which we are about to define. The same applies to nurses and to the doctor's specialized auxiliaries, who are adequately trained for their occupation, in similar yet differentiated fashion.

What then is medical training for disaster situations? In this problem, the fundamental rules of any medical training and the essential principles of teaching in general must serve as a guide to reflection and achievement.

The essential purpose of teaching is to train. This it achieves by developing the pupil's capacity for thought and observation, by instilling in him the habit of working on his own to improve his knowledge; in short by training his mind and equipping him with a method of work. These are essential factors in education and are equally necessary for training for medical practice in disasters, but they concern rather the method followed, general considerations and conclusions, and reflections on study motivation. Training also leads to special scientific and practical knowledge, and this is where training for medical practice in disaster situations becomes decidedly more specific.

In my opinion, the first fundamental requirement is to provide training for the work to be done, bearing in mind the starting point, that is to say the level of instruction of all grades of personnel who are to be trained, and clearly to fix the aim pursued, namely the qualifications which should be obtained by students of various categories. The work to be done should therefore be defined beforehand. It might vary depending upon the organization of civil defence services, and on the medical practice in disaster situations within those services which each country or region decides to set up. Teaching should vary according to those differing conditions.

It follows that a large number of first-aiders will be needed during the first stages of relief operations. They should do their utmost to save life, by maintaining or restoring the state known as consciousness, breathing, and blood circulation. They should enable casualties to survive until a doctor can arrive on the scene, or ensure that the patient is fit for conveyance to the doctor; they should in each case discern the degree of urgency of first aid; they should distinguish between patients who may go home after an initial dressing, pending subsequent treatment as outpatients, and those who are dying and are, alas, beyond medical help. 
As many first-aid workers as possible should be trained. The more competent should, besides rendering the usual first aid, be able to give injections of analgesic or resuscitating substances, or administer blood transfusions or blood substitutes, on instructions and under supervision. Nowadays we know that no medical education is valid without adequate psychological training. In preparing for medical practice in disaster situations, of which the requirements contrary to current ethics have a traumatic effect on the mind, special psychological training must be provided and must be suited to the special aspects of the care which is to be given.

As many doctors as possible will then be needed for treatment at the preliminary stage. They will do no more than the first-aiders, but where the latter fail they will resort to methods which only a doctor can apply. They will check on the care given by first-aiders in the more difficult or complex cases. They may revise evacuation priorities. The first aid which they render the patients brought to them, being more complete and lasting, will enable all to wait a few hours for second-stage treatment. Doctors will also be better able to prognosticate the death of those for whom further care can do no more than relieve pain. This activity, as we have outlined it here, clearly indicates the fairly simple yet effective and highly productive training which many doctors should receive. It should not prove difficult to train doctors, whatever their age, whether specialists or general practitioners, to give such first-stage care. A short theoretical and practical course should suffice.

Doctors will also be needed for second-stage care. This, in my opinion, is the most important problem in such training and the crucial point of medical activity in case of disaster, because there should be enough doctors to treat a large number of patients in case of need. At that stage, it would be necessary to apply current methods in surgery or in certain specialties fully enough for the treatment to be determinate. It should be possible, too, to treat cases of poisoning or infection and the medical sequelae of shock, so as not to encumber hospitals equipped for intensive and specialized treatment. This would imply genuine surgical training coupled with general medical training.

This brings us directly to another problem, that of the number of doctors available. In countries where the technical development 
of surgery and medicine has not reached the highest peak, the situation would appear to be more favourable in this respect. It may sound like a paradox: but in the most important hospitals, the advanced surgery practised is dependent on very specialized instruments and methods, and some of the surgeons might, despite their great proficiency in their own specialty, find themselves somewhat handicapped in administering second-stage care to multiple-injury patients. It is true that " retraining" would be a rapid and easy process, for it would only be a matter of extending their practical knowledge, which at their level would soon be assimilated, without increasing their qualifications through prolonged training. But where is there today such a surplus of highly qualified surgeons as to allow a large number of them to be used for second-stage care without dangerously depleting hospitals providing third-stage care?

Surgeons in local, district or regional hospitals, who in a number of countries are excellent and have lost nothing of their medical versatility, would be the most suitable medical personnel for extra training, being easily adaptable to that stage. However, in many countries there are too few surgeons, so that one may wonder whether the requirements of medicine in disaster situations and the training it presupposes should not be accompanied by some professional reorganization, with the clearsighted co-operation of medical associations. One may besides rightly consider that that reorganization would be for the benefit not only of the medical profession-provided it were supplied with enough adequate local hospitals-but of the population as well.

At this second stage, the doctor's auxiliaries should be nurses for general care and a few specialized nursing personnel (operating theatre nurses, anaesthetists, etc.). They would be selected from among the current personnel at that level and should, in principle, require no special training. As they are very scarce everywhere, while potential first-aid workers are, in principle, as numerous as the whole of the population, it would appear that the teaching of medicine in disaster situations should enable some of the more qualified first-aiders to rise to the level of auxiliary nurses for second-stage care.

If we agree that such second-stage care should be administered mainly by civil defence services (for it is hard to say where else 
it could be given on an adequate scale), we can see the burden of teaching they will be called upon to bear. Logically, I might say that if the civil defence services were to put 50 per cent of their emergency hospitals into permanent use (and since they are standing empty pending some hypothetical disaster, they represent a large amount of idle capital, and deteriorate for want of use) they would automatically maintain an adequate corps of physicians and provide possibilities of work which they would have neither at home nor in the more specialized hospitals to which they do not belong. The task of teaching would thus be eased.

Lastly, doctors and all types of auxiliaries would be needed for third-stage care. This does not differ from the determinate and specialized care administered in the country at all times and therefore does not presuppose any special teaching. We may therefore exclude it from the various aspects of our problem. Customary medical training does provide such personnel. The sudden steep rise in the number of patients requiring third-stage care following a disaster poses not so much the problem of training medical personnel as the problem of organization, involving the arrival of a sufficient number of medical reinforcements from neighbouring or more distant areas not affected by the disaster, or the removal of patients to areas where there is still room for their treatment.

From the foregoing we may conclude that the teaching of medicine in disaster situations should, in the first place, provide special general training for all doctors, for male and female nurses, specialized auxiliaries and first-aiders whose task it is to administer first and second-stage care. In addition to the principles of organization and the general principles of medicine in disaster situations, which should be taught by way of introduction with illustrations and the necessary explanatory material, all medical personnel will have to learn, or re-learn, some therapeutic acts that are simple, rapid and effective in life-saving, namely:

1. Haemostasis.

2. Revival techniques.

3. Immobilizing fractures to permit transport.

4. Preparing the patient for removal. 
5. First protective dressing, with adequate antisepsis, and the cleaning of wounds.

6. The covering of burns pending the patient's removal to a centre providing specialized care.

7. Pain-reducing methods.

8. Pre-, per- and post-operative care of patients.

9. Screening for admission to treatment centre, followed by screening within the centre, and finally removal from the centre.

10. Familiarity with use and maintenance of equipment and installations for treatment (which should be standardized at the various levels and stages of treatment).

Such training should include a number of hours of theoretical courses, with ample use of audio-visual methods (to make up for certain pupils' lack of imagination or to convey an impression of reality and thereby ensure psychological training). This should be followed by some hours of practical training with mock patients. The first-aiders should if possible be afforded an opportunity, at least for one day, to see patients in an accident emergency department, or to accompany first-aid workers of a permanent ambulance service on their rounds for one or two days. Doctors should spend at least a few days in such a service.

Teaching should as far as possible be simple and reduced to essentials. Therapy should be split up and arranged in a series of operations in order that they may be repeated until they become automatic like a reflex. This offers the greatest likelihood of such acts being performed adequately despite rush, excitement or fatigue. Such automatic performance should also free part of the faculties of attention and reflection, which should always maintain their maximum power. A fundamental course on a two-tier basis, one level for first-aid workers and the other for doctors, should be followed by all persons required to care for disaster victims; on their doing so depend continuity of care and unity of action.

In addition, technical courses should be provided for male and female nurses and for specialized auxiliaries who are not professionals and whom it is proposed to train with civil defence services for levels at which second-stage care is given. Nurses' training colleges should make their teaching methods and experience available.

Again, doctors selected for second-stage care should receive fur- 
ther surgical and medical training where there are not enough general practitioners to be assigned to civil defence emergency hospitals. Such theoretical and practical courses can only be held in hospitals, with the co-operation of the medical and surgical emergency services, the emergency services of various specialties, and the services of accident surgery and of locomotor system surgery.

The training acquired must be kept up. Yearly refresher courses, briefer than the training courses, should be attended by all first-aid workers who are not professional nurses and by doctors whose customary activities do not correspond to first- and second-stage care (according to the post to which they are assigned in case of disaster). Refresher courses should include a personal entrance examination to ascertain what has been retained of the earlier training and to adapt the refresher courses to individual requirements. The courses should consist of theoretical revision with copious examples and suitable practical exercises. Finally, the spirit of research and further learning should be encouraged among medical personnel.

In conclusion, we may agree that we know, in principle, what medical training for disaster situations should be, and how it should be organized. Yet it is a heavy and complex task. There is no certainty that the means will be available in every country, and in any case the availability of such means is likely to be gradual everywhere. It is satisfying for the mind and soothing to the conscience to embark on such work at this juncture, in a general well-defined context, and gradually to build up the various parts. If necessary, the best possible use will be made of whatever means are devised, progressively with their availability. If the work is based on a sensible and adequate plan, time can only lead to success. As we have seen, the total development of such teaching must necessarily lead to some public health organization and to considerably increased facilities for the care of masses of casualties in the wake of a disaster of any kind. 